\title{
EFFECTIVENESS OF NEONATAL EMERGENCY TRANSPORT SERVICE AT DIFFERENT GESTATIONAL AGE: AN EPIDEMIOLOGICAL STUDY IN LAZIO REGION
}

\author{
F. Crescenzi ${ }^{1}$, M. Gente ${ }^{2}$, D. Di Lallo ${ }^{3}$, F. Franco ${ }^{3}$, F. Landolfo ${ }^{1}$, O. Di Lella ${ }^{2}$, G. Guasticchi ${ }^{3}$, on behalf of \\ Neonatal Emergency Transport Service Lazio Region, Rome Italy \\ ${ }^{1}$ Medical and Surgical Neonatology, Children Hospital Bambino Gesù, ${ }^{2}$ Neonatal Emergencency Transport \\ Service, 'La Sapienza, ${ }^{3}$ Agency for Public Health-Lazio Region, Roma, Italy
}

Background and aims: Neonatal transport is a key component of neonatal-perinatal care. The aim of the study is to evaluate the performance of the care provided by Neonatal Emergency Transport Service (NETS), using the Transport Risk Index of Physiologic Stability score (TRIPS) for different groups of Gestational Age (GA).

Methods: We studied 758 newborns with non-surgical diseases who were transferred from birth- hospital to the 13 Neonatal Intensive Care Units of Lazio region within 72 hours of birth, between may 2009 and june 2010. Pre and post TRIPS score was collected by a dedicated informative system maintained by NETS. We stratified the analysis on TRIPS for four GA groups (22-26, 27-31, 32-36, $\geq 37$ weeks).

Results: The mean gestational age was 35 week (SD 4.1). The mean pre and post TRIPS score was, respectively, 25.5 and 25.5 at 22-26wks, 14.2 and 13.2 at 27-31wks, 5.6 and 5.0 at 32-36wks and 6.2 and 5.8 at $\geq 37 \mathrm{wks}$. There was a significant improvement between pre and post TRIPS score based on Wilcoxon mached paired signed-ranks test $(\mathrm{p}<0,001)$. The stratified analysis by GA groups confirmed the overall results for all GA groups except for GA 22-26wks.

Conclusions: In a geographical area with a high rate of neonatal transfer $(2 \%)$, our study demonstrates a good performance of an organized neonatal transport service, more evident in infants with GA over 26 weeks. These results must be better evaluated taking into account mortality and morbidity outcomes and costs. 